\title{
Nanotube-based gas sensors - role of structural defects
}

J. Andzelm, N. Govind, A. Maiti

May 10, 2005

Chemical Physics Letters 
This document was prepared as an account of work sponsored by an agency of the United States Government. Neither the United States Government nor the University of California nor any of their employees, makes any warranty, express or implied, or assumes any legal liability or responsibility for the accuracy, completeness, or usefulness of any information, apparatus, product, or process disclosed, or represents that its use would not infringe privately owned rights. Reference herein to any specific commercial product, process, or service by trade name, trademark, manufacturer, or otherwise, does not necessarily constitute or imply its endorsement, recommendation, or favoring by the United States Government or the University of California. The views and opinions of authors expressed herein do not necessarily state or reflect those of the United States Government or the University of California, and shall not be used for advertising or product endorsement purposes. 


\title{
Nanotube-based gas sensors - role of structural defects
}

\author{
Jan Andzelm, ${ }^{1,}$ Niranjan Govind, ${ }^{1}$ and Amitesh Maiti ${ }^{2, *}$ \\ ${ }^{1}$ Accelrys Inc., 10188 Telesis Court, San Diego, CA 92121 \\ ${ }^{2}$ Lawrence Livermore National Lab, University of California, Livermore, CA 94551
}

\begin{abstract}
Existing theoretical literature suggests that defect-free, pristine carbon nanotubes (CNTs) interact weakly with many gas molecules like $\mathrm{H}_{2} \mathrm{O}, \mathrm{CO}, \mathrm{NH}_{3}, \mathrm{H}_{2}$, and so on. The case of $\mathrm{NH}_{3}$ is particularly intriguing because this is in disagreement with experimentally observed changes in electrical conductance of CNTs upon exposure to these gases. In order to explain such discrepancy, we have carried out Density Functional Theory (DFT) investigations of the role of common atomistic defects in CNT (Stone-Wales, monovacancy, and interstitial) on the chemisorption of $\mathrm{NH}_{3}$. Computed binding energies, charge transfer, dissociation barriers, and vibrational modes are compared with existing experimental results on electrical conductance, thermal desorption and infrared spectroscopy.
\end{abstract}

PACS: 61.46.+w, 68.43.-h, 73.22.-f, 85.35.-p

Keywords: Nanotubes, sensors, IR, chemisorption

${ }^{\S}$ Present address: Army Research Lab, Aberdeen Proving Ground, MD 21010

${ }^{*}$ Corresponding author, E-mail: maiti2@llnl.gov 
Of all the projected application areas of Nanotechnology, gas sensors based on CNTs and nanowires appear to be the ones closest to commercial reality. Detection of gas molecules such as $\mathrm{NO}_{2}, \mathrm{O}_{2}, \mathrm{NH}_{3}, \mathrm{~N}_{2}$, $\mathrm{CO}_{2}, \mathrm{H}_{2}, \mathrm{CH}_{4}, \mathrm{CO}$, or even water is important for monitoring environmental, medical or industrial conditions. Recently, it was reported [1-4] that semiconducting single wall nanotubes (s-SWNT) could detect, at room temperature, small concentrations of $\mathrm{NH}_{3}$ and $\mathrm{NO}_{2}$ with high sensitivity. The detection is done by measuring changes in the nanotube conductance upon exposure to the gases. Thus the measured electrical conductance at room temperature increases by three orders of magnitude upon exposure to 200 ppm of $\mathrm{NO}_{2}$, and decreases by two orders of magnitude upon exposure to $1 \%$ of $\mathrm{NH}_{3}$. Exposure to $\mathrm{O}_{2}$ also appears to consistently increase the electrical conductance [5], although the effect is not as dramatic as for $\mathrm{NO}_{2}$.

Considering the fact that the s-SWNT used in the experiment of ref. [1] was effectively $p$-type (i.e. holes were the majority carriers), the observed behavior of conductance changes can be easily rationalized based on a simple charge transfer model between the adsorbed molecule and the SWNT. Thus $\mathrm{NO}_{2}$, as a Lewis acid, can withdraw electrons from s-SWNT thus increasing the hole-population and enhancing conductance. $\mathrm{NH}_{3}$ on the other hand is a Lewis base that can donate electrons to the s-SWNT thereby depleting the hole-population and SWNT conductance. However, such conclusion is based on the assumption that the molecule has a stable adsorbed structure on the SWNT at room temperature, which would be possible if the SWNT-molecule binding is strong enough. For $\mathrm{NO}_{2}$ one could explain such stability through the formation of $\mathrm{NO}_{3}[6]$, a common feature observed on metal-oxide surfaces [7] and nanowires as well [8]. However, several calculations for $\mathrm{NH}_{3}$ have yielded only very weak binding and very small charge transfer [2, 9-11]. This is in disagreement not only with the observed drop in electrical conductance at room temperature, but also with recent temperature-programmed-desorption (TPD) measurements [12], which shows that most of the gas desorbs only at elevated temperatures (500-700 K).

A logical resolution to the above problem would be to note that SWNTs are likely to have some defects incorporated either thermally or during high-temperature growth conditions. Also, they are not in isolation, and surrounding environment (oxygen, water vapor), or the substrate, or metal contacts at the ends might directly or indirectly provide a mechanism of binding of the gas molecules. Limited 
investigations have been made on the effect of metal contacts, especially in connection with $\mathrm{O}_{2}$ adsorption $[13,14]$. However, $\mathrm{NH}_{3}$ adsorption appears to occur along the whole length of the tube [1], which suggests either topological defects on the tube itself, or an indirect mechanism due to involvement of solvents or the substrate.

In this Letter, we investigate the role of three types of topological defects commonly considered for SWNTs and graphene sheet, i.e., a Stone-Wales (SW) defect [15] (i.e. a 5775 defect formed by bondrotation), a monovacancy (i.e. missing a C-atom), and an insterstitial (i.e. an extra bridging C). The presence of such geometric defects has recently been confirmed experimentally [16]. As a concrete model of s-SWNT, we chose the $(8,0)$ SWNT. All the defect and chemisorbed structures were created by suitably modifying the original defect-free tube (Fig. 1a). In order to speed up our calculations the initial structures were minimized with the COMPASS forcefield [17], followed by further relaxation with the DFT code $\operatorname{DMol}^{3}[18,19]$. Within $\mathrm{DMol}^{3}$, we performed all-electron calculations on a "medium" integration grid, with the electronic wave functions expanded in a double-numeric polarized (DNP) basis set truncated at a real-space cutoff of $4.5 \AA$. A nonlocal DFT Hamiltonian was used with gradient-corrected exchangecorrelation functional due to Perdew, Burke, and Ernzerhof [20], while accurate Brillouin zone sampling was ensured by summing over a finite set of K-points chosen according to the Monkhorst-Pack scheme [21] with a grid spacing of $0.05 \AA^{-1}$. Accurate transition state barriers were obtained by a generalized synchronous transit method recently extended to periodic supercells [22]. Charge transfer to and from the nanotube and was obtained through computing the partial charge on each chemisorbed atom by Hirshfeld population analysis [23].

Fig. 1 illustrates four types of basic defects considered in this work: (i) SW1, a non-chiral Stone-Wales (SW) defect (Fig. 1d); (ii) SW2, a chiral Stone-Wales defect (Fig. 1f); (iii) a monovacancy (V) (Fig. 1b); and (iv) an interstitial (I) (Fig. 1c). It also considers the situation in which an $\mathrm{O}_{2}$ molecule has dissociated over a SW1 (Fig. 1e) [24]. Table 1 lists the respective DMol ${ }^{3}$-computed formation energies of these defects for supercells of two sizes, i.e., two and four times the smallest periodic segment of the $(8,0)$ SWNT, with sufficient vacuum left in the transverse directions in order to ensure minimal interaction between translational images of SWNTs. The resulting orthorhombic supercells have dimensions of $8.45(16.9)$ x 20 
x $20 \AA^{3}$, with the SWNT aligned along the x-axis, consist of a total of 64 (128) C-atoms for the original clean tube, and are henceforth referred to simply as the shorter (longer) supercell respectively. From Table 1, we note that SW1 is energetically more stable than SW2, and is therefore the only SW defect considered for chemisorption reactions below. It is also clear that $\mathrm{V}$ and especially I are more localized defects and the shorter supercell would suffice to investigate them. SW1, on the other hand, should require the longer supercell to obtain size-independent properties. We have chosen our supercells accordingly in all calculations reported below.

For each defect, i.e., V, I and SW1, we first computed the binding energies of $\mathrm{NH}_{3}$ in a physisorbed state. As in ref. [9], all the physisorbed structures were weakly bound, with very small charge transfer to the adsorbed molecule. It was tempting, therefore, to investigate chemisorbed structures, which could potentially involve higher binding and charge transfer. Chemisorption of $\mathrm{NH}_{3}$ typically involves dissociation into $\mathrm{NH}_{2}$ and $\mathrm{H}$ fragments, each forming chemical bonds to substrate atoms.

Table 2 displays reaction energetics $\left(\Delta \mathrm{E}_{\text {react }}\right)$ and activation barrier $\left(\Delta \mathrm{E}_{\text {act }}\right)$ for such dissociation reaction on pure $(8,0)$ tube, as well as the four types of defects $\mathrm{V}, \mathrm{I}, \mathrm{SW} 1$, and $\mathrm{O}_{2}$ pre-dissociated on SW1 (henceforth referred to as SW1_O_O). The main results could be summarized as follows: (i) dissociation of $\mathrm{NH}_{3}$ on a defect-free tube is an endothermic process with a significant barrier, and therefore unlikely even at elevated temperatures; (ii) at both $\mathrm{V}$ and I the dissociation becomes exothermic with energy gains of 2.49 and $2.26 \mathrm{eV}$ respectively. The activation barrier for dissociation is rather low at $\mathrm{V}$, and should happen readily at room temperature, while that at I is also possible, although at a slower rate; (iii) at SW1 the dissociation is marginally exothermic, and the activation barrier is lower than that for a defect-free tube, although a bit high for chemisorption to happen readily at room temperatures. However, the presence of pre-dissociated $\mathrm{O}$ (SW1_O_O) significantly enhances the stability of chemisorbed $\mathrm{NH}_{3}$ and makes the $\mathrm{NH}_{3}$ dissociation process nearly spontaneous; (iv) in all cases there is net electron transfer from the chemisorbed $\mathrm{NH}_{3}$ to the s-SWNT. As compared to the defect-free tube, the amount of charge transfer to $\mathrm{V}$ increases almost two-and-a-half fold, while that to SW1_O_O is enhanced nearly seven-fold. It is also to be noted here that multiple $\mathrm{O}_{2}$ molecules could potentially dissociate on the same SW1 defect [24], thus providing dissociation sites for multiple $\mathrm{NH}_{3}$ molecules, leading to much higher net charge transfer to the SWNT. 
Large charge transfer should qualitatively explain the observed drops in electrical conductance [1], although a quantitative comparison would require explicit electronic transport calculations. Such an effort using a non-equilibrium Green's Function (NEGF) approach is currently underway [25].

Finally, we computed the infrared (IR) spectra of various chemisorbed structures of Fig. 2 in order to compare with recent FTIR observations [12]. We used the IR/Raman module [26] and the COMPASS forcefield [17] from Accelrys. Fig. 3 displays the IR spectra of three systems: $\mathrm{NH}_{3}$ chemisorbed at a vacancy (Fig. 2b), $\mathrm{NH}_{3}$ chemisorbed at $\mathrm{SW} 1$ _O_O (Fig. 2e), and an isolated $\mathrm{NH}_{3}$ molecule [27]. Isolated $\mathrm{NH}_{3}$ molecule displays four peaks: $\mathrm{A}$ - an "umbrella"-like symmetric bend at $952 \mathrm{~cm}^{-1}, \mathrm{C}$ - two degenerate asymmetric bend modes at $1624 \mathrm{~cm}^{-1}, \mathrm{E}-\mathrm{a}$ symmetric $\mathrm{N}-\mathrm{H}$ stretch at $3337 \mathrm{~cm}^{-1}$, and $\mathrm{F}$ - two degenerate asymmetric stretch modes at $3448 \mathrm{~cm}^{-1}$. The frequencies of these modes agree well with experimental values for ammonia [12] justifying our use of the COMPASS forcefield in the computation of IR. As Fig. 3 indicates, mode A gets red-shifted by $\sim 100 \mathrm{~cm}^{-1}$ in the spectrum of Fig. 2, and blue-shifted by $\sim 50 \mathrm{~cm}^{-1}$ in the spectrum of Fig. 2e. Frequencies of modes C, E and F, on the other hand, essentially coincide in all the three spectra, except for a slight blue shift of mode $C$ for Fig. $2 b$. In addition, the spectrum of Fig. $2 b$ has a mode E corresponding to $\mathrm{C}-\mathrm{H}$ stretch, and the spectrum of Fig. 2e has two extra modes: B - corresponding to HOC-angle bend, and G - corresponding to OH stretch. Experimental FTIR spectra of ref. [12] in the range 1000-3500 $\mathrm{cm}^{-1}$ includes features around $\mathrm{B}, \mathrm{C}$, and a wide peak covering $\mathrm{D}, \mathrm{E}$ and $\mathrm{F}$, although the peak around B was interpreted as a heavily-blue-shifted mode A [12].

In summary, we have investigated the effect of commonly observed topological defects on ammonia sensing properties of a semiconducting SWNT. Defects like vacancies and Stone-Wales defect with predissociated oxygen are found to spontaneously chemisorb $\mathrm{NH}_{3}$ into dissociated $\mathrm{NH}_{2}$ and $\mathrm{H}$ fragments. Computed binding energies are consistent with TDP results that elevated temperatures are necessary to drive out all of the adsorbed ammonia, significant electron transfer from adsorbates explain large drops in electrical conductance observed experimentally, while computed IR spectra provide useful interpretation of recently measured FTIR data. 
Acknowledgment: We would all like to thank Accelrys Inc., and Jan Andzelm would particularly like to thank Fujitsu Ltd. for the support of this work. The work at LLNL was performed under the auspices of the U.S. Department of Energy by the University of California Lawrence Livermore National Laboratory under Contract W-7405-Eng-48. 
References:

1. J.Kong, N.R.Franklin, C.Zhou, M.G.Chapline, S.Peng, K.Cho and H.Dai, Science 287, 622 (2000).

2. P. Qi, O. Vermesh, M.Grecu, A. Javey, Q. Wang, H.Dai, S. Peng, K.Cho, Nano Lett. 3, 347 (2003).

3. L. Valentini, I. Armentano, J.M. Kenny, C.Cantalini, L.Lozzi and S.Santucci, Appl. Phys.Lett. 82, $961(2003)$.

4. S. Chopra, A.Pham, J. Gaillard, A.Parker and M.Rao, Appl.Phys. Lett.80, 4632 (2002).

5. P.G.Collins, K.Bradley, M.Ishigami and A. Zettl, Science 287, 1801 (2000).

6. S. Peng, K. J. Cho, P. Qi and H. Dai, Chem. Phys. Lett. 387, 271 (2004).

7. J. A. Rodriguez, T. Jirsak, G. Liu, J. Dvorak, and A. Maiti, J. Am. Chem. Soc. 123, 9597 (2001).

8. A. Maiti, J. A. Rodriguez, M. Law, P. Kung, J. McKinney and P. Yang, Nano Letters 3, 1025 (2003).

9. C. W. Bauschlicher and A. Ricca, Phys. Rev. B 70, 115409 (2004).

10. H.Chang. J.D.Lee, S.M.Lee and Y.H.Lee, Appl. Phys. Lett. 79, 3863 (2001).

11. J. Zhao, a. Buldum, J. Han and J.P. Lu, Nanotechnology 13, 195 (2002).

12. M.D. Ellison, M.J.Crotty, D. Koh, R.L. Spray and K.E. Tate, J. Phys. Chem. B 108, 7938 (2004). See Fig. 1 and Table 1 of this reference for FTIR data and information on vibrational frequencies.

13. S. Heinze at al., Phys. Rev. Lett. 89, 106801 (2002).

14. T. Yamada, Phys. Rev. B 69, 125408 (2004). 
15. A.J. Stone and D.J. Wales, Chem.Phys.Lett. 128, 501 (1986).

16. A. Hashimoto, K.Suenaga, A. Gloter, K. Urita and S. Lijima, Nature, 430, 870 (2004).

17. H. Sun, J. Phys. Chem. B 102, 7338 (1998).

18. See Accelrys webpage: $\underline{h t t p: / / w w w . a c c e l r y s . c o m / m s t u d i o / m s ~ m o d e l i n g / d m o l 3 . h t m l . ~}$

19. B. Delley, J. Chem. Phys. 92, 508 (1990).

20. J. P. Perdew, K. Burke, M. Ernzerhof, Phys. Rev. Lett. 77, 3865 (1996).

21. H. J. Monkhorst, J. D. Pack, Phys. Rev. B 13, 5188 (1976).

22. N. Govind, M. Petersen, G. Fitzgerald, D. King-Smith and J. Andzelm, Comp. Mat. Sci. 28, 250 (2003).

23. F. L. Hirshfeld, Theoret. Chim. Acta 44, 129 (1977).

24. We started from an initial geometry in which a spin-triplet $\mathrm{O}_{2}$ molecule was physisorbed on a SW1 defect and then dissociated into bridging positions on various pairs of bonds. In most cases, the barrier for $\mathrm{O}_{2}$ dissociation was around or less than $1 \mathrm{eV}$, and the structure upon oxygen incorporation was energetically more stable than the physisorbed geometry (i.e. formation energy was negative, see numbers in parentheses in the last row of Table 1). Among all our investigated structures, Fig. 1e appears to incur the lowest $\mathrm{O}_{2}$ dissociation barrier, of only $\sim 0.6 \mathrm{eV}$. Once dissociated, the O-atoms can diffuse to neighboring bonds, with migration energy barriers ranging from $0.5-1.2 \mathrm{eV}$. It is clearly possible to have multiple $\mathrm{O}_{2}$ molecules dissociated at the same SW1.

25. A. Maiti, H. Mehrez, A. Svizhenko, and M. P. Anantram, in progress.

26. See Accelrys webpage: http://www.accelrys.com/products/datasheets/c2_ir_raman_us_data.pdf.

27. This is essentially the same spectrum one would obtain for $\mathrm{NH}_{3}$ weakly physisorbed on a SWNT because the IR contribution from an all-C structure like SWNT is very small. 
Table 1. Formation energies of various defects on a $(8,0)$ SWNT computed with respect to a defect-free tube. For SW1_O_O (last row) the formation energy is computed with respect to a defect-free tube $+\mathrm{O}_{2}$ molecule (spin triplet).

\begin{tabular}{|l|l|l|}
\hline Defect type & $\begin{array}{l}\text { Formation energy (eV) } \\
\text { (shorter tube) }\end{array}$ & $\begin{array}{l}\text { Formation energy (eV) } \\
\text { (longer tube) }\end{array}$ \\
\hline $\begin{array}{l}\text { Straight Stone-Wales (SW1) } \\
\text { (Fig. 1b) }\end{array}$ & 2.58 & 1.86 \\
\hline $\begin{array}{l}\text { Chiral Stone-Wales (SW2) } \\
\text { (Fig. 1f) }\end{array}$ & 4.16 & 3.56 \\
\hline $\begin{array}{l}\text { Vacancy (V) } \\
\text { (Fig. 1c) }\end{array}$ & 6.21 & 6.07 \\
\hline $\begin{array}{l}\text { Interstitial (I) } \\
\text { (Fig. 1d) }\end{array}$ & 5.43 & 5.30 \\
\hline $\begin{array}{l}\text { O2 dissociated on SW1 } \\
\text { (SW1_O_O) (Fig. 1e) }\end{array}$ & $1.71\left(-0.87^{*}\right)$ & $1.44\left(-0.42^{*}\right)$ \\
\hline
\end{tabular}

*computed w.r.t. a $(8,0)$ tube with a SW1 defect + isolated $\mathrm{O}_{2}$ molecule (spin triplet) 
Table 2. $\mathrm{NH}_{3}$ dissociation (into $\mathrm{NH}_{2}$ and $\left.\mathrm{H}\right)$ on a $(8,0) \mathrm{SWNT}$ : reaction energetics $\left(\Delta \mathrm{E}_{\text {reac }}\right)$, activation barrier $\left(\Delta \mathrm{E}_{\mathrm{act}}\right)$, and net electron transfer $(\Delta \mathrm{q})$ from $\mathrm{NH}_{2}$ and $\mathrm{H}$ groups to the SWNT. Negative values of $\left(\Delta \mathrm{E}_{\text {reac }}\right)$ denotes an exothermic process.

\begin{tabular}{|c|c|c|c|c|}
\hline Substrate & $\begin{array}{l}\text { Resulting bonding configuration } \\
\text { on SWNT surface }\end{array}$ & $\begin{array}{l}\Delta \mathrm{E}_{\text {reac }} \\
(\mathrm{eV})\end{array}$ & $\begin{array}{l}\Delta \mathrm{E}_{\text {act }} \\
(\mathrm{eV})\end{array}$ & $\begin{array}{l}\Delta \mathrm{q} \text { from } \mathrm{NH}_{3} \\
\text { (el) }\end{array}$ \\
\hline SWNT (defect-free) & $\mathrm{C}_{3}-\mathrm{NH}_{2}+\mathrm{C}_{3}-\mathrm{H}$ (Fig. 2a) & +0.77 & 2.38 & 0.025 \\
\hline $\mathrm{V}$ & $\mathrm{C}_{2}-\mathrm{NH}_{2}+\mathrm{C}_{2}-\mathrm{H}$ (Fig. 2b) & -2.49 & 0.35 & 0.063 \\
\hline I & $\mathrm{NH}_{2}-\mathrm{C}_{2, \text { bridge }}-\mathrm{H}$ (Fig. 2c) & -2.26 & 1.13 & 0.036 \\
\hline SW1 & $\mathrm{C}_{3,577}-\mathrm{NH}_{2}+\mathrm{C}_{3,577}-\mathrm{H}$ (Fig. 2d) & -0.17 & 1.50 & 0.044 \\
\hline SW1_O_O & 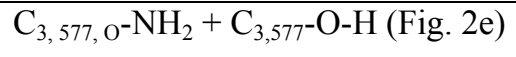 & -2.77 & 0.25 & 0.176 \\
\hline
\end{tabular}

$\mathrm{C}_{3}=$ Regular 3-fold coordinated $\mathrm{sp}^{2}$ carbon on a defect-free SWNT; $\mathrm{C}_{2}=\mathrm{C}_{3}$ atom with a missing Cneighbor; $\mathrm{C}_{3,577}=\mathrm{sp}^{2}$ carbon at a SW1 site shared by two heptagons and a pentagon; $\mathrm{C}_{3,577, \mathrm{O}}=\mathrm{C}_{3,577}$ atom with a bridging $\mathrm{O}$ separating it from one of its $\mathrm{C}$ neighbors 


\section{Figure captions:}

Fig. 1. Various structural defects on a $(8,0)$ nanotube: (a) defect-free tube; (b) vacancy (V) (c) interstitial (I); (d) straight Stone-Wales defect (SW1); (e) $\mathrm{O}_{2}$ molecule pre-dissociated at SW1 (O_O_SW1); (f) slanted Stone-Wales defect (SW2). Atoms in the vicinity of the defects are shown in stick representation.

Fig. 2. $\mathrm{NH}_{3}$ dissociated at defects of Figs. 1(a-e). Dissociated $\mathrm{NH}_{2}$ and $\mathrm{H}$ fragments are shown in ball representation. In Fig. 2e, the second $\mathrm{O}$ breaks an $\mathrm{O}-\mathrm{C}$ bond and creates a $\mathrm{OH}$ group single-bonded to the other C-atom.

Fig. 3. Computed Infrared (IR) spectra using the COMPASS forcefield. Three spectra are displayed: $\mathrm{NH}_{3}$ weakly physisorbed on SWNT (solid line with filled diamonds); $\mathrm{NH}_{3}$ chemisorbed at a vacancy (open circles); and $\mathrm{NH}_{3}$ chemisorbed at $\mathrm{SW} 1{ }_{-} \mathrm{O} \_\mathrm{O}$ (dotted line). The first spectrum corresponds to that of a free $\mathrm{NH}_{3}$ molecule, while the last two spectra correspond to structures in Fig. $2 \mathrm{~b}$ and 2e respectively. Important IR peaks, denoted by letters A-G, are discussed in the text. 


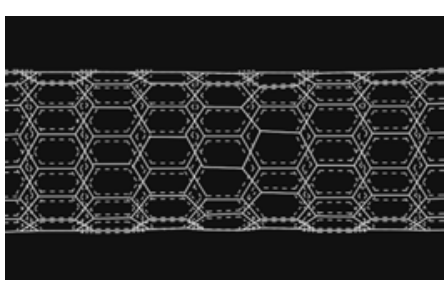

(a)

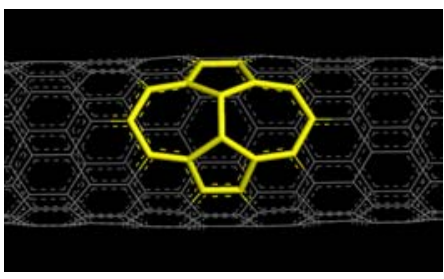

(d)

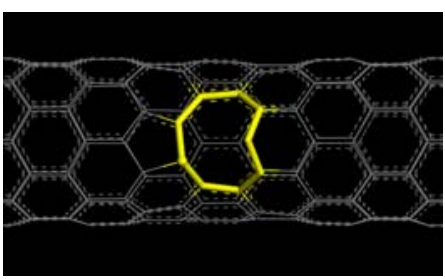

(b)

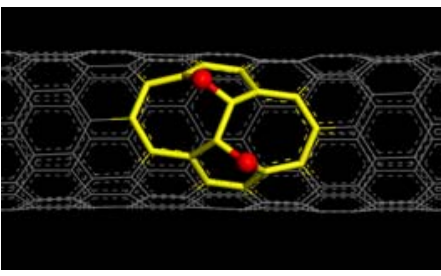

(e)

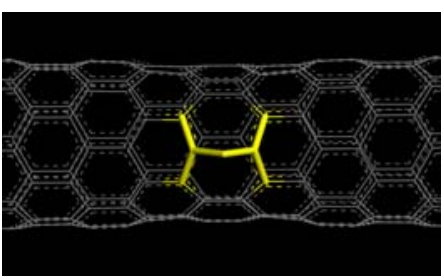

(c)

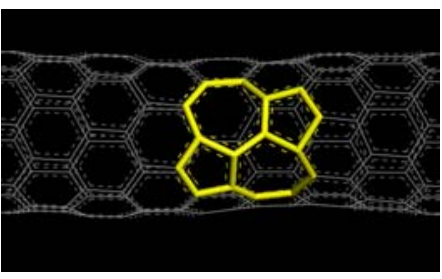

(f)

Figure 1 


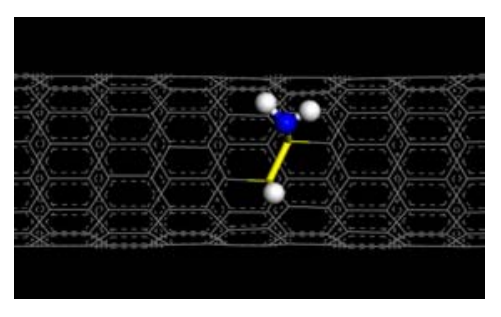

(a)

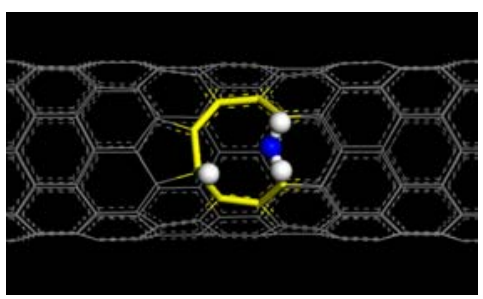

(b)

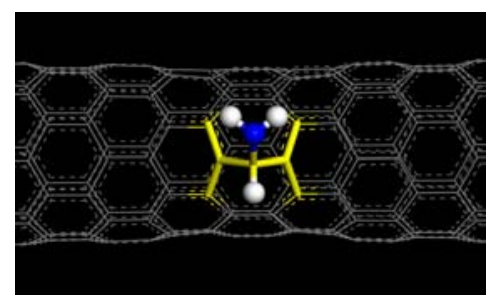

(c)

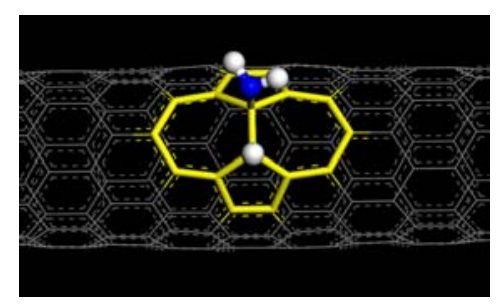

(d)

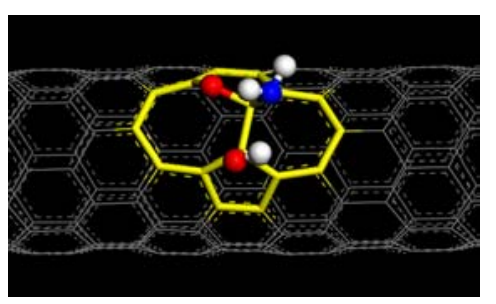

(e)

Figure 2 


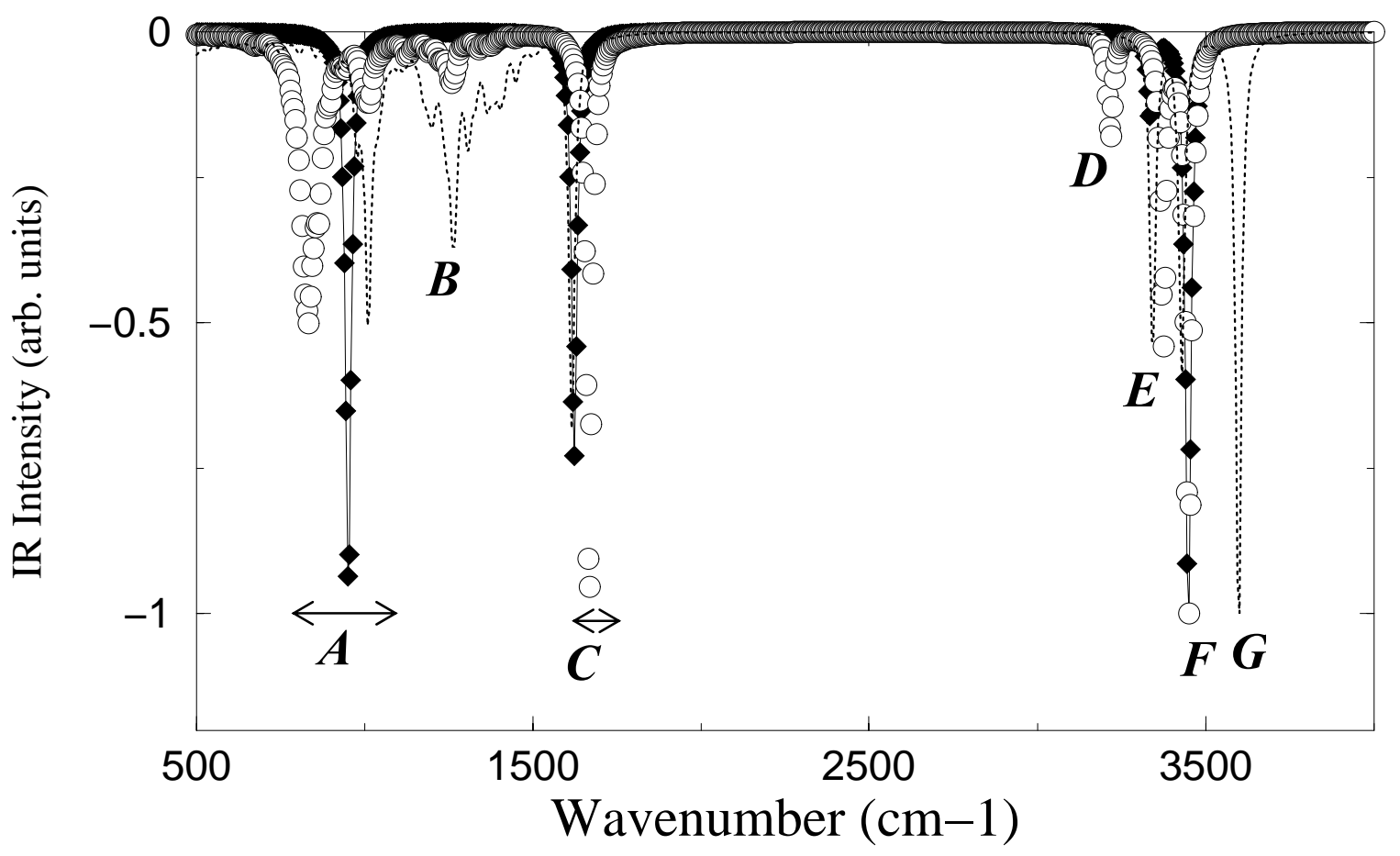

Figure 3 